\title{
"Stopping before you start": Reducing and preventing initiation of tobacco use in the ACT
}

\author{
$\underline{\text { L Freebairn }}^{\text {a,b }}$, K Kreuger ${ }^{\text {c }}$, C Watson ${ }^{\text {d }, ~ Z ~ Y a n g ~ a, ~ A ~ P r o d a n ~}{ }^{\text {e,b }}$, K.I. Graham ${ }^{\text {a }}$, G Draper ${ }^{\text {a }}$, \\ G McDonnell $^{\mathrm{f}}$, E Nixon ${ }^{\mathrm{a}}$, and J-A Atkinson ${ }^{\mathrm{f}, \mathrm{g}, \mathrm{h}}$ \\ ${ }^{a}$ Preventive and Population Health, Health Directorate, Canberra, ACT, Australia, ${ }^{b}$ The Australian \\ Prevention Partnership Centre, Sax Institute, Sydney, NSW, Australia, ${ }^{c}$ Kreuger Consulting LLC, Austin, \\ Texas, USA, ${ }^{d}$ Public Health Protection and Regulation, Health Directorate, Canberra, ACT, Australia, \\ ${ }^{e}$ Engineering \& Mathematics, Western Sydney University, Sydney, NSW, Australia, ${ }^{f}$ Decision Analytics, \\ Sax Institute, Sydney, NSW, Australia, g.Translational Health Research Institute, Western Sydney \\ University, Sydney, NSW, Australia, ${ }^{h}$ Sydney School of Public Health, University of Sydney, Sydney, \\ NSW, Australia \\ Email: louise.freebairn@act.gov.au)
}

\begin{abstract}
Tobacco is the leading cause of preventable death in Australia and contributes to 5.4\% of disease burden in the Australian Capital Territory. Initiation of tobacco use is most likely to occur during adolescence and young adulthood (at less than 20 years). Prevention of tobacco initiation involves a combination of regulatory, educational and health promotion interventions including restrictions on the sale of tobacco products. This paper reports on the development and use of an agent-based model to explore the impact of modifying three hypothetical regulatory and health promotion interventions: 1) increasing the minimum purchasing age for tobacco products, 2) reducing retail sales of tobacco products to persons under the minimum purchasing age and 3) reducing secondary sharing of tobacco products to persons under the minimum purchasing age using health promotion messaging. The model was built using a participatory approach that engaged policy officers, health promotion officers, epidemiologists, biostatisticians and computer scientists. The structure of the model included interacting state chart representations of smoking and level of concern about tobacco use (engagement status) and a pro-smoking score, which defined the hazard rate of initiation, cessation, and relapse. The pro-smoking score was a function of several risk factors including engagement, social effect of having more or fewer smoking peers, addiction and withdrawal levels and access to tobacco products. Parameterisation of the model drew on a range of data sources with local data being prioritised where it was available. A series of scenarios comparing the impact of the interventions on smoking prevalence rates and age of initiation are reported. Of the three interventions simulated, increasing the minimum purchasing age from 18 to 21 years had the greatest impact on smoking prevalence across the population, reducing the prevalence of smoking from $8.5 \%(95 \%$ CI $7.8,9.2)$ to $6.9 \%(95 \%$ CI $6.4,7.4)$ five years post-intervention and $4.1 \%(95 \%$ CI 3.8, 4.3) 20 years post intervention (Figure 1). The interventions aimed to reduce the sale of tobacco products to minors and reduce secondary sharing produced small reductions on their own. However,
\end{abstract} when implemented in combination with increasing the minimum purchasing age, they significantly increased the impact of this intervention from ten years postimplementation, ultimately resulting in a prevalence rate of $2.8 \%(95 \%$ CI 2.6, 3.0) 20 years post-implementation. Given the challenges associated with ceasing tobacco use, these in silico experiments demonstrate the importance of regulatory public health interventions to delay, and therefore potentially prevent initiation.

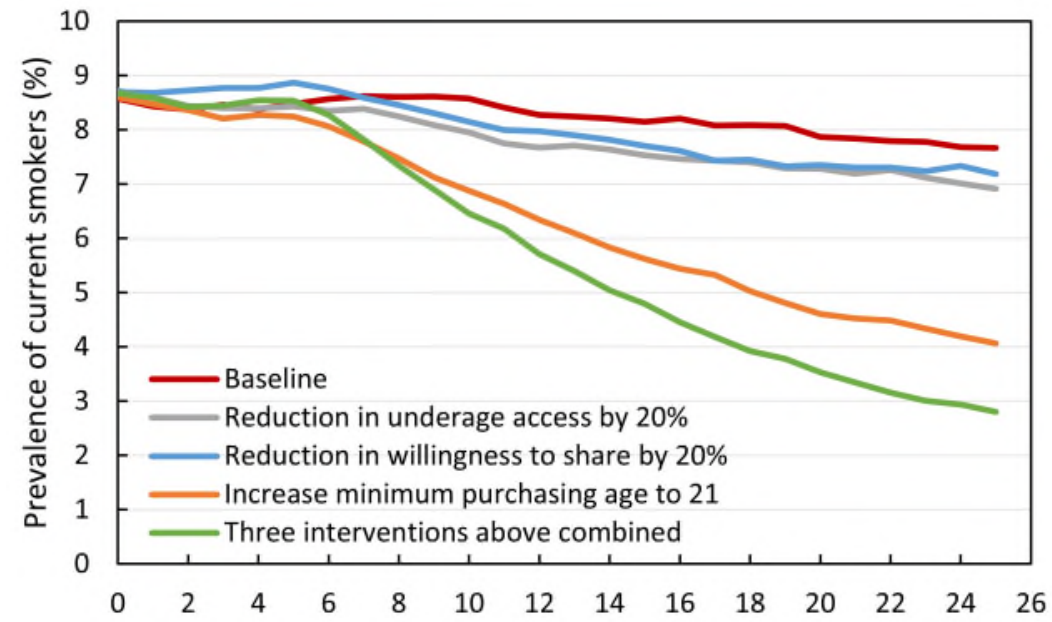

Figure 1. Simulated change in prevalence of current smokers for intervention strategies. Interventions start at year 5 .

Keywords: Tobacco use, health modelling, agent-based 


\section{INTRODUCTION}

Tobacco smoking is the leading cause of preventable death in Australia, contributing to $41 \%$ of respiratory diseases, $22 \%$ of cancers, $11.5 \%$ of cardiovascular diseases and $3.7 \%$ of endocrine disorders (Australian Institute of Health and Welfare 2019). Tobacco use contributed to 5.4\% of the total disease burden in the Australian Capital Territory (ACT) in 2011 (Australian Institute of Health and Welfare 2017). The rate of daily tobacco smoking in Australia has halved since 1991, but since 2013 this decline has plateaued, and, in 2016, $12.2 \%$ of Australians still identified as daily smokers. The reduction in smoking rates is predominantly related to young people not initiating smoking, rather than people quitting smoking. The ACT has the lowest adult daily smoking rate of all Australian states and territories at 9.9\%, but this rate has not changed since 2013 (Australian Institute of Health and Welfare 2017).

There are many influences for initiation of smoking by adolescents; the key ones being family and peer influence, poor academic performance, alcohol use, and stress/anxiety/depression (O'Loughlin, Karp et al. 2009, Leventhal, Urman et al. 2017). Adolescents have been found to be more susceptible to nicotine addiction than adults and start to experience symptoms of nicotine addiction at levels as low as one or two exposures (O'Loughlin, DiFranza et al. 2003). Over two-thirds of people who try one cigarette become daily smokers, at least temporarily (Birge, Duffy et al. 2018). Age at initiation of smoking is a significant factor for continuation of smoking. Men aged 35 or over, who had started smoking under the age of 16 had an odds ratio of 2.1 for not quitting smoking compared to those who had started after age 19 (Khuder, Dayal et al. 1999).

All Australian states and territories currently ban sales of tobacco to people under the age of 18. Enforcement of bans on sales to youths has been found to be effective in reducing youth uptake of smoking in Australia (Tutt, Bauer et al. 2009). In the United States, at least 16 states have now introduced a 21-year age limit for purchasing tobacco and there is some evidence that this limit reduces access to tobacco by 15-17-year old adolescents who often access tobacco through their 18-year old peers (Kessel Schneider, Buka et al. 2016). Despite current access restrictions, 48\% of Australian smokers aged 12-17 report accessing cigarettes from friends, while $15 \%$ ask other people to purchase for them, $18 \%$ buy them directly and $17 \%$ are given, or take cigarettes from family members (Guerin and White 2018).

Systems science methods such as dynamic simulation modelling can provide decision makers with insights into the causal mechanisms underlying human behaviour and decision making that we see in the real world (Hassmiller Lich, Ginexi et al. 2013, Marshall and Galea 2015). Modelling allows decision makers to explore the potential impact of policy interventions, including hypothetical and locally untested interventions, before they are implemented in their local context (Sterman 2006, Marshall, Burgos-Liz et al. 2016, Freebairn, Atkinson et al. 2018). Advances in modelling software are increasing model transparency, making them more accessible to non-modellers (Osgood 2017). This facilitates the active involvement of expert stakeholders in the model development process, increasing the opportunities for interdisciplinary learning about the focus issue and building trust in the model outputs (Hovmand 2014, Voinov, Kolagani et al. 2016, Freebairn, Rychetnik et al. 2017, Freebairn, Atkinson et al. 2018). Agent-based modelling has been identified as a useful method to explore strategies to prevent tobacco use (Wallace, Geller et al. 2015) and a previous agent-based modelling study of tobacco use demonstrated the impact of retail density on purchasing behaviour (Luke, Hammond et al. 2017). The aim of this study was to develop a dynamic simulation model, with policy and program implementers, that explored the social and behavioural dynamics underlying initiation and use of tobacco by using agent-based modelling methods. Intervention scenarios explored the impact of raising the minimum age of purchasing tobacco products, social marketing to decrease secondary supply of tobacco and reducing the retail sale of tobacco products to young people under the minimum purchasing age.

\section{METHODS}

\subsection{Model development}

The model development process drew on best practice guidelines for computational modelling and included the grounding of assumptions in theory and evidence, sensitivity testing and calibration (Marshall, Burgos-Liz et al. 2015, Wallace, Geller et al. 2015). The model was built using a participatory approach that engaged policy officers, health promotion officers, epidemiologists, biostatisticians and computer scientists. The model was constructed using AnyLogic simulation software (http://www.anylogic.com/).

\subsection{Model inputs and data sources}

The structure and parameterisation of the model drew on a range of data sources, including census and population data, systematic reviews, meta-analyses, accepted formulas and conceptual models, survey data, policy/program effectiveness data, economic data and the expert knowledge of the multidisciplinary stakeholders who participated in model development. Local data was prioritised where this was available. 
Freebairn et al., "Stopping before you start": Reducing and preventing initiation of tobacco use in the ACT

Expert knowledge was leveraged when other evidence options were exhausted. Census, population and health system data were sourced from the Australian Bureau of Statistics, Australian Institute of Health and Welfare and ACT Health administrative data collections.

\subsection{Model structure}

This agent-based model includes interacting state chart representations of smoking and engagement status.

Smoking status: The primary behaviour of agents (people) in the model is smoking, captured in the smoking statechart. The 3 main states are: Never Smoker, Current Smoker, and Ex-Smoker. Persons become Current Smokers through the init_self rate transition, and they make quit attempts through the quit rate transition. Relapses into smoking occur according to the relapseShort or relapseLong rate transitions. This model examined access to tobacco, especially for underage persons, therefore never smokers were also classified as "Has Access" and "No Access": persons in the No Access state could not transition to CurrentSmoker.

Engagement status: The engagement statechart represents a level of concern for the health effects of tobacco use. It is defined to have four engagement states: Unconcerned, Concerned, Engaged, and Disengaged. Agents have an annual probability to become concerned proportional to age, reflecting the assumption that younger agents are less likely to be concerned about the negative outcomes of smoking behaviour. Agents who are concerned and also current-smokers move probabilistically through a cycle of concern-engagementdisengagement-concern, spending on average about 2 months in each state. This reflects the fact that current smokers report quit attempts about twice per year (Borland, Partos et al. 2012).

\subsection{Behaviour Rules}

Pro-smoking Score: The Person agent uses a pro-smoking score to determine transition hazards in the Smoking statechart. A positive value means the person has a positive perception of smoking, while a negative value means a negative perception. The pro-smoking bias is determined using 5 separate personal characteristics, as shown in equation 1 for the pro-smoking score, $\boldsymbol{P}$.

$$
P=\beta_{0}\left(\beta_{E} E+\beta_{S} S+\beta_{R} R+\beta_{A} A+\beta_{W} W\right)
$$

Equation variables are Engagement, Social effect, Rebelliousness, Addiction, and, Withdrawal and their corresponding beta terms. The value in the brackets lies between -1 and 1 , and $\beta_{0}$ (a model parameter) is used to convert $P$ into a hazard value. It is calculated in the function updateSmokingHazards. For a Never Smoker, a positive pro-smoking bias determines the initiation hazard, while a negative pro-smoking score sets the initiation hazard to 0 . As a Current Smoker, a negative value determines the quit hazard (0 otherwise), and as an Ex-Smoker, a positive value sets both relapse hazards (0 otherwise).

The Engagement statechart determines the value of $E$ by associating each state with a score, from -1 to 1 . The Unconcerned state corresponds to an agent having no engagement bias, therefore $E=0$. Concerned agents show a slightly anti-smoking engagement bias where $E=-0.1$. Agents who are engaged have a strong desire to quit, and therefore a strongly anti-smoking engagement bias with $E=-1$. Finally, to capture the idea that failure to quit while engaged can lead to a period during which a smoker resigns themselves to their addiction, disengaged smokers have a strongly pro-smoking engagement bias $(E=1)$. As noted above, never-smokers can be unconcerned or concerned, therefore engagement itself does not increase the probability of initiating.

The Social effect is proportional to the percentage of socially connected peers who are smokers. Someone with a social network of $0 \%$ current smokers will have a social effect score of -1 (strongly anti-smoking), 50\% current smokers receive a score of 0 (neutral), and $100 \%$ results in a score of 1 (strongly pro-smoking), with linear interpolation. Since the only other factor that can cause initiation is the social effect, rebelliousness is required to allow some persons to be the first smoker in their social network. Each person is given an intrinsic rebelliousness, drawn from an exponential distribution parameterised so that only a few agents have sufficient rebelliousness to initiate of their own accord. It is combined with a person's age such that agents achieve peak rebelliousness at 18 years of age, increasing linearly from 15 and decreasing linearly to 21 .

Addiction and withdrawal determine the values for $A$ and $W$, respectively. Addiction and withdrawal are positive numbers (between 0 and 1) calculated from two interacting temporal difference learning equations (Hammond, Ornstein et al. 2012). The logic was defined to replicate assumptions on the dynamics of addiction and withdrawal. Addiction, we assume, starts at 0 for never-smokers, and gradually increases upon initiation of cigarette smoking behaviour. The rate of increase is highest at first, and gradually reduces to 0 when the 
agent reaches a maximum, stable, addiction level. ${ }^{1}$ After a quit attempt, it gradually reduces to a minimum value somewhat above 0 , replicating the assumption that the relapse of a long-term former-smoker is more likely than initiation by a comparable never-smoker (Caraballo, Kruger et al. 2014).

Withdrawal is captured separately because it only plays a role upon cessation. When a quit attempt is made, it should quickly increase to a peak value, after which it gradually reduces to near 0 , representative of the fact that most relapses occur within relatively short periods of time following smoking cessation (Hughes 2007). The height of the withdrawal peak is controlled by the level of addiction reached at the moment of cessation.

Social Network: Persons in this model are connected in a social network. Each agent is connected bidirectionally with a number of other agents. Social connections play two roles in this model. Firstly, as discussed above, an agent's pro-smoking bias is influenced by the smoking behaviour of their social peers. Secondly, underage smokers may require someone in their social network who can provide them with tobacco. Therefore, social connections are assumed to represent only close friends, since only close friends are assumed to influence the pro-smoking bias. A person's weaker social connections will primarily influence their tobacco access, which is captured through the social distance of sharing, discussed below.

This network is constructed using Social Circles (Hamill and Gilbert 2010), which specifies that persons should connect with others based on their location in a 2D social environment. This allows specific network features known to exist in human social networks, such as network clustering, and the small world effect (Hamill and Gilbert 2010). However, this model adds the ability to capture age similarity; the idea that persons are more likely to connect to other persons similar to them in age. ${ }^{2}$ The network is formed by creating a reach for each person. This is parameterised to have, on average, 50 others in its catchment. This list of 50 persons is then filtered by similarity in age and each person connects with the most similar agents to themselves. The parameter connectionsMadePerAgent determines the number of connections made (Hamill and Gilbert 2010).

Access: Two types of access to tobacco are represented: primary and secondary. Primary access is where a person can purchase cigarettes on their own. They may have reached the minimum purchasing age, or they have access to a retailer who will sell them tobacco even though they are underage. The probability of an underage person having access is determined using the Main variable primaryUnderageAccessAgeProportions, which divides the underage population into 3 age groups and assigns each a probability based on unpublished compliance audits of retail compliance by age in the ACT. Secondary access occurs through persons with primary access sharing tobacco. Individuals with primary access can obtain tobacco products for others who do not have primary access, giving those persons secondary access. Persons can pass along access only if they are current-smokers, and their friends are close enough to the person with primary access (within a number of steps as specified by the accessDepth model parameter).

\subsection{Interventions tested in scenarios}

This model has several possible interventions, all triggered at a specific time in the model in order to allow sufficient time for the model to achieve stability.

Reducing retail sales to underage persons: This intervention focused on reducing retail sales of tobacco products to underage persons. The model assumed that underage persons have a probability of successfully purchasing tobacco products, depending on their age. This intervention reduces that probability by a number ranging from 0 to 1 . For example, if a person aged 17 has a 50\% chance of having underage primary access, enabling this intervention with a strength of 0.5 reduces that to a $25 \%$ chance.

Media Campaign to reduce secondary supply: This supposes a media campaign that is capable of convincing persons who have primary access to not share cigarettes with underage persons. Therefore, this reduces the number of agents who are willing to share. The strength of this intervention is a number from 0 to 1 . Setting it to 0.5 , for example, reduces the default probability to be willing from 0.5 to 0.25 .

Minimum Purchase Age: This intervention increases the minimum purchasing age from 18. The intervention age is specified by a model parameter, normally set to 18 . When this intervention is triggered, it increases the purchasing age by 1 year per model-year until the desired minimum purchasing age is reached.

\footnotetext{
${ }^{1}$ The maximum addiction level is assumed to be different between agents to replicate the fact that different people appear to have different susceptibilities to tobacco addiction. It ranges across the population according to a triangular distribution from 0.4 to 1.0 , with the model parameter peakSusceptibility specifying the peak.

${ }^{2}$ According to Hamill and Gilbert, human social networks have clustering coefficients in the range of approximately 0.25 to 0.5 . Even with the changes to the Social Circles model, clustering coefficients in this model are within these bounds.
} 


\subsection{Validation and Parameter Estimation}

This model generated a simulated population in which policy experiments could be conducted. Therefore, during model construction, the interdisciplinary group observed outputs of the model, both aggregate and for different typical agents, and highlighted any apparent discrepancy. While qualitative, this face-validation process was effective at catching inappropriate behavioural assumptions at an early stage.

Model parameters were estimated to match aggregate consumption patterns reflecting features of the Australian context. The model has 11 parameters which could not be obtained from data, previous studies, or expert opinion, due in large part to the detail of individual human behaviour included in this model. Therefore, for these parameters, we sampled estimates in the non-intervention case according to a Nearly Orthogonal Latin Hypercube (NOLH) design (Sanchez and Wan 2015). The main outcome measure was the population prevalence of smoking. Of the 50 total parameter combinations generated by the NOLH design, we selected the 5 closest for each of 3 prevalence groups: $10 \%, 20 \%$, and 30\%, as these ranges describe appropriate ranges for different sectors of the Australian population. For the analysis presented in this paper, the lowest prevalence group was chosen to most closely reflect the overall ACT smoking prevalence rate (9.5\%) (Australian Institute of Health and Welfare 2017). The model used a warm-up period of 90 model-years (approximately 1 agentlifetime), and the model ran for 25 model-years, with any intervention taking place at model-year 5.

\subsection{Data Analysis}

Outputs from the model were summarised using the $\mathrm{R}$ statistical package to obtain means, standard errors and 95\% confidence intervals; summary data was tabulated and graphed in Microsoft Excel. Following experimentation,ten runs were deemed sufficient to account for stochasticity, provide stable predictions of scenario performance and of the variance in performance while balancing the time required to run each scenario. 95\% confidence intervals about the means were reported as estimates of the variation between simulation runs. The comparison of simulation results between baseline and intervention scenarios was expressed as a per cent difference in prevalence of current smokers. Statistical significance for differences between baseline and intervention scenarios was determined using t-test.

\section{RESULTS}

The scenario with the greatest impact on smoking prevalence (proportion of current smokers in the population) was increasing the minimum purchasing age from 18 to 21 years. This intervention significantly decreased smoking prevalence within five years of implementation from 8.2\% (95\% CI 7.7, 8.8) at implementation to $6.9 \%(95 \%$ CI 6.4, 7.4) five years post- and 4.1\% (95\% CI 3.8, 4.3) 20 years post-implementation (Table 1).

The interventions aiming to reducing retail sales to underage persons by $20 \%$ and social media campaigns aiming to reduce the secondary sharing of tobacco products to underage persons by $20 \%$ resulted in small reductions in smoking prevalence independently (Table 1). However, when combined with increasing the minimum purchasing age, they increased the intervention effectiveness significantly, reducing smoking prevalence to $4.8 \%(95 \% \mathrm{CI} 4.4,5.2) 10$ years post-, and $2.8 \%$ (95\% CI 2.6, 3.0) 20 years post-implementation.

Table 1. Simulated change in prevalence of current smokers for different intervention strategies. Interventions start at year five.

\begin{tabular}{cccccc}
\hline \multirow{2}{*}{$\begin{array}{c}\text { Model } \\
\text { Time } \\
\text { (years) }\end{array}$} & Baseline & \begin{tabular}{c} 
Reduction in \\
underage access by \\
\cline { 2 - 6 }
\end{tabular} & $\begin{array}{c}\text { Prevalence of Current Smokers (95\% CI) } \\
\text { willingness to share } \\
\text { by 20\% }\end{array}$ & $\begin{array}{c}\text { Increase minimum } \\
\text { purchasing age to } 21\end{array}$ & $\begin{array}{c}\text { Three } \\
\text { interventions } \\
\text { combined }\end{array}$ \\
\hline 0 & $8.6(8.2,9.0)$ & $8.7(8.3,9.1)$ & $8.7(8.0,9.4)$ & $8.6(8.1,9.1)$ & $8.7(8.0,9.4)$ \\
5 & $8.5(7.8,9.2)$ & $8.4(7.8,9.0)$ & $8.9(8.2,9.5)$ & $8.2(7.7,8.8)$ & $8.5(7.9,9.2)$ \\
10 & $8.6(7.9,9.2)$ & $8.0(7.4,8.5)$ & $8.1(7.7,8.6)$ & $6.9(6.4,7.4)^{*}$ & $6.5(6.0,6.9)^{*}$ \\
15 & $8.1(7.5,8.8)$ & $7.5(7.0,8.0)$ & $7.7(7.1,8.3)$ & $5.6(5.3,6.0)^{*}$ & $4.8(4.4,5.2)^{*}$ \\
20 & $7.9(7.2,8.5)$ & $7.3(6.9,7.6)$ & $7.3(6.9,7.8)$ & $4.6(4.3,4.9)^{*}$ & $3.5(3.2,3.8)^{*}$ \\
25 & $7.7(7.1,8.2)$ & $6.9(6.5,7.4)^{*}$ & $7.2(6.7,7.6)$ & $4.1(3.8,4.3)^{*}$ & $2.8(2.6,3.0)^{*}$ \\
\hline * Indicating significant difference from the baseline $(\mathrm{p}<0.05)$ & & &
\end{tabular}

* Indicating significant difference from the baseline $(\mathrm{p}<0.05)$.

\section{DISCUSSION}

The simulation experiments reported here prioritised scenario testing of three interventions aimed at preventing initiation of tobacco use in young people. The regulatory intervention of increasing the minimum purchasing age from 18 to 21 years was found to be most effective in these in silico experiments. Interventions aimed at decreasing illegal sales and secondary supply of tobacco products to persons under the minimum purchasing age did not significantly decrease smoking prevalence, however when used in combination with increasing the minimum purchasing age, did significantly increase the impact of the purchasing age intervention. 
Most people who try a cigarette for the first time are aged under 18 years (AIHW, 2017). A New Zealand study found a strong age-related decline in initiation of tobacco use with 14.2\% of 15-17 year olds initiating smoking compared with $3 \%$ of adults aged 20 to 24 years and approximately $1 \%$ for those 25 years and older (Maubach, Hoek et al. 2013). Daily smoking in adolescent smokers is a risk factor that may be particularly important in the development of nicotine dependence and the progression to sustained smoking. Recent (past 30-day) smoking among adolescents, inclusive of non-daily smoking, was a strong predictor of future smoking behaviour in adulthood (Dutra and Glantz 2018). As a result, tobacco control efforts to reduce initiation of smoking have often targeted young people in the hope of producing a generation of never smokers (Elders, Perry et al. 1994).

Ninety per cent of Australian adult smokers report that they regret taking up smoking (Nayak, Pechacek et al. 2017). Quitting is difficult, with approximately $40 \%$ of smokers trying to quit each year, with an average of one quit attempt made every 6 months (Borland, Partos et al. 2012). Many smokers make 30 or more quit attempts before being successful (Chaiton, Diemert et al. 2016). The findings of this study therefore make a compelling case for regulatory interventions to prevent smoking initiation in young people to reduce the risk of lifetime smoking and its sequelae.

\section{CONCLUSIONS AND RECOMMENDATIONS}

The public health interventions aimed at reducing initiation of smoking in young people were effective in reducing smoking prevalence in this simulated agent population, particularly when used in combination. Three hypothetical interventions were tested in these scenarios: 1) increasing the minimum purchasing age from 18 years to 21 years, 2) reducing the sale of tobacco products to persons under the minimum purchasing age and 3 ) reducing secondary sharing of tobacco products to underage persons. Increasing the minimum purchasing age was found to be most effective, with no significant reductions in smoking prevalence achieved through the reduction of sales of tobacco products to of secondary sharing with minors. These findings are important for public health policy as delaying the initiation of tobacco use beyond adolescence is likely to prevent smoking behaviour overall due to the low initiation rate in adulthood (Edwards, Carter et al. 2013).

The scenarios presented here are based on the underlying smoking prevalence of the ACT community (approximately 10\%). Previous agent-based modelling studies have found that intervention effectiveness varies according to the characteristics of the community within which they are implemented (Luke, Hammond et al. 2017). Future applications of the model include further exploration of the impact of the intervention scenarios on sub-populations with higher smoking prevalence rates. A third intervention option will explore the impact of public health education campaign to discourage secondary sharing of tobacco products with young people. An important focus for future modelling research will be the uptake and long-term use of e-cigarettes.

\section{ACKNOWLEDGEMENTS}

The interventions tested in these simulations were hypothetical and do not reflect any current or planned policy directions of ACT Health or the ACT Government.

\section{REFERENCES}

Australian Institute of Health and Welfare (2017). National Drug Strategy Household Survey 2016: detailed findings. Drug Statistics Canberra, Australian Institute of Health and Welfare: 168.

Australian Institute of Health and Welfare (2019). Australian Burden of Disease Study: impact and causes of illness and death in Australia 2015. Austalian Burden of Disease. Canberra, Australian Institute of Health and Welfare: 212.

Birge, M., S. Duffy, J. A. Miler and P. Hajek (2018). "What Proportion of People Who Try One Cigarette Become Daily Smokers? A Meta-Analysis of Representative Surveys." Nicotine Tob Res 20(12): 1427-1433.

Borland, R., T. R. Partos, H. H. Yong, K. M. Cummings and A. Hyland (2012). "How much unsuccessful quitting activity is going on among adult smokers? Data from the International Tobacco Control Four Country cohort survey." Addiction 107(3): 673-682.

Caraballo, R. S., J. Kruger, K. Asman, L. Pederson, R. Widome, C. I. Kiefe, B. Hitsman and D. R. Jacobs, Jr. (2014). "Relapse among cigarette smokers: the CARDIA longitudinal study - 1985-2011." Addictive behaviors 39(1): 101-106.

Chaiton, M., L. Diemert, J. E. Cohen, S. J. Bondy, P. Selby, A. Philipneri and R. Schwartz (2016). "Estimating the number of quit attempts it takes to quit smoking successfully in a longitudinal cohort of smokers." $\underline{B M J}$ Open 6(6): e011045.

Dutra, L. M. and S. A. Glantz (2018). "Thirty-day smoking in adolescence is a strong predictor of smoking in young adulthood." Prev Med 109: 17-21.

Edwards, R., K. Carter, J. Peace and T. Blakely (2013). "An examination of smoking initiation rates by age: results from a large longitudinal study in New Zealand." Aust N Z J Public Health 37(6): 516-519. 
Freebairn et al., "Stopping before you start": Reducing and preventing initiation of tobacco use in the ACT

Elders, M. J., C. L. Perry, M. P. Eriksen and G. A. Giovino (1994). "The report of the Surgeon General: preventing tobacco use among young people." Am J Public Health 84(4): 543-547.

Freebairn, L., J.-A. Atkinson, P. M. Kelly, G. McDonnell and L. Rychetnik (2018). "Decision makers' experience of participatory dynamic simulation modelling: methods for public health policy." BMC Medical Informatics and Decision Making 18(1): 131.

Freebairn, L., L. Rychetnik, J.-A. Atkinson, P. Kelly, G. McDonnell, N. Roberts, C. Whittall and S. Redman (2017). "Knowledge mobilisation for policy development: implementing systems approaches through participatory dynamic simulation modelling." Health research policy and systems 15(1): 83.

Guerin, N. and V. White (2018). ASSAD 2017 Statistics \& Trends: Australian Secondary Students' Use of Tobacco, Alcohol, Over-the-counter Drugs, and Illicit Substances. Melbourne, Cancer Council Victoria: 86.

Hamill, L. and N. Gilbert (2010). "Simulating large social networks in agent-based models: A social circle model." Emergence: Complexity and Organization 12(4): 78-94.

Hammond, R. A., J. T. Ornstein, L. K. Fellows, L. Dube, R. Levitan and A. Dagher (2012). "A model of food reward learning with dynamic reward exposure." Front Comput Neurosci 6: 82.

Hassmiller Lich, K., E. Ginexi, M. , N. Osgood, D. and P. Mabry, L. (2013). "A Call to Address Complexity in Prevention Science Research." Prevention Science 14(3): 279-289.

Hovmand, P. S. (2014). Community Based System Dynamics. New York, United States of America, Springer.

Hughes, J. R. (2007). "Effects of abstinence from tobacco: valid symptoms and time course." Nicotine Tob Res 9(3): 315-327.

Kessel Schneider, S., S. L. Buka, K. Dash, J. P. Winickoff and L. O'Donnell (2016). "Community reductions in youth smoking after raising the minimum tobacco sales age to 21." Tob Control 25(3): 355-359.

Khuder, S. A., H. H. Dayal and A. B. Mutgi (1999). "Age at smoking onset and its effect on smoking cessation." Addictive Behaviors 24(5): 673-677.

Leventhal, A. M., R. Urman, J. L. Barrington-Trimis, N. I. Goldenson, K. Gallegos, C. P. Chou, K. Wang, K. Berhane, T. B. Cruz, M. A. Pentz, J. Unger and R. S. McConnell (2017). "Perceived stress and poly-tobacco product use across adolescence: Patterns of association and gender differences." J Psychiatr Res 94: 172-179.

Luke, D. A., R. A. Hammond, T. Combs, A. Sorg, M. Kasman, A. Mack-Crane, K. M. Ribisl and L. Henriksen (2017). "Tobacco Town: Computational Modeling of Policy Options to Reduce Tobacco Retailer Density." American Journal of Public Health 107(5): 740-746.

Marshall, B. D. L. and S. Galea (2015). "Formalizing the Role of Agent-Based Modeling in Causal Inference and Epidemiology." American Journal of Epidemiology 181(2): 92-99.

Marshall, D. A., L. Burgos-Liz, M. J. Ijzerman, N. D. Osgood, W. V. Padula, M. K. Higashi, P. K. Wong, K. S. Pasupathy and W. Crown (2015). "Applying dynamic simulation modeling methods in health care delivery research-the SIMULATE checklist: report of the ISPOR simulation modeling emerging good practices task force." Value in health : the journal of the International Society for Pharmacoeconomics and Outcomes Research 18(1): 5-16.

Marshall, D. A., L. Burgos-Liz, K. S. Pasupathy, W. V. Padula, M. J. Ijzerman, P. K. Wong, M. K. Higashi, J. Engbers, S. Wiebe, W. Crown and N. D. Osgood (2016). "Transforming Healthcare Delivery: Integrating Dynamic Simulation Modelling and Big Data in Health Economics and Outcomes Research." PharmacoEconomics 34(2): 115-126.

Maubach, N., J. A. Hoek, R. Edwards, H. Gifford, S. Erick and R. Newcombe (2013). "'The times are changing': New Zealand smokers' perceptions of the tobacco endgame." Tob Control 22(6): 395-400.

Nayak, P., T. F. Pechacek, P. Slovic and M. P. Eriksen (2017). "Regretting Ever Starting to Smoke: Results from a 2014 National Survey." Int J Environ Res Public Health 14(4).

O'Loughlin, J., J. DiFranza, R. F. Tyndale, G. Meshefedjian, E. McMillan-Davey, P. B. Clarke, J. Hanley and G. Paradis (2003). "Nicotine-dependence symptoms are associated with smoking frequency in adolescents." Am J Prev Med 25(3): 219-225.

O'Loughlin, J., I. Karp, T. Koulis, G. Paradis and J. Difranza (2009). "Determinants of first puff and daily cigarette smoking in adolescents." Am J Epidemiol 170(5): 585-597.

Osgood, N. (2017). Frontiers in Health Modeling. Systems Science and Population Health. A. El-Sayed and S. Galea. Oxford, United Kingdom, Oxford University Press: 191.

Sanchez, S. M. and H. Wan (2015). Work smarter, not harder: A tutorial on designing and conducting simulation experiments. 2015 Winter Simulation Conference, Huntington Beach, California USA, IEEE.

Sterman, J. D. (2006). "Learning from evidence in a complex world." American journal of public health 96(3): 505-514.

Tutt, D., L. Bauer and J. Difranza (2009). "Restricting the retail supply of tobacco to minors." J Public Health Policy 30(1): 68-82.

Voinov, A., N. Kolagani, M. K. McCall, P. D. Glynn, M. E. Kragt, F. O. Ostermann, S. A. Pierce and P. Ramu (2016). "Modelling with stakeholders -Next generation." Environmental Modelling \& Software 77: $196-220$.

Wallace, R., A. Geller and V. A. Ogawa (2015). "Assessing the use of agent-based models for tobacco regulation." Assessing the use of agent-based models for tobacco regulation. 